\title{
Groin pain syndrome: an association of different pathologies and a case presentation
}

\author{
Gian Nicola Bisciotti ${ }^{1}$ \\ Alessio Auci ${ }^{2}$ \\ Francesco Di Marzo 3 \\ Roberto Galli ${ }^{4}$ \\ Luca Pulici ${ }^{5}$ \\ Giulia Carimati 5 \\ Alessandro Quaglia ${ }^{5}$ \\ Piero Volpi ${ }^{5}$
}

1 Kinemove Rehabilitation Center Pontremoli, Italy

2 Dipartimento di Diagnostica Ospedaliera, Radiodagnostica, ASL1 Massa-Carrara, Italy

3 General Surgery Unit, Ospedale di Pontremoli, Italy

4 Department of Health Science, Postgraduate School in Occupational Medicine, University of Genoa San Martino, Genoa, Italy

5 Department of Knee Orthopedic and Sports Traumatology Unit, Humanitas Research Hospital, Rozzano, Italy

\section{Corresponding author:}

\section{Piero Volpi}

Department of Knee Orthopedic and Sports Traumatology Unit, Humanitas Research Hospital

Via Manzoni 56

20089 Rozzano, Italy

E-mail: piero.volpi@humanitas.it

\section{Summary}

Background: groin pain affects all types of athletes, especially soccer players. Many diseases with different etiologies may cause groin pain.

Purpose: offer a mini review of groin pain in soccer accompanied by the presentation of a case report highlighting the possible association of more clinical frameworks into the onset of groin pain syndrome, in order to recommend that clinical evaluations take into account possible associations between bone, muscle and tendon such as inguinal canal disease.

Conclusion: the multifactorial etiology of groin pain syndrome needs to be examined with a comprehensive approach, with standardized clinical evaluation based on an imaging protocol in order to evaluate all possible diseases.

Study design: Mini review- Case report (Level V).

KEY WORDS: groin pain syndrome, sport hernia, osteitis pubis, adductor tendinopathy.

\section{Introduction}

Groin pain (GP) is a widely known issue among professional and amateur athletes. It is very important to specify that "groin pain" means "pain in the groin area" and is not a diagnosis. According to the different injuries and the different symptoms referred by the patient we can identify different kinds of groin pain, with as many different causes.

One of the most rational GP classification is proposed by Omar et $\mathrm{al}^{1}$. In this classification, diagnosis is based on 37 major diseases grouped into 10 categories (Tab. 1).

Furthermore it is important to note also the conclusions reached by the Groin Pain Consensus Conference 2014 during which were defined the four major sub headings of GP in athletes that are 2 :

i. common musculoskeletal clinical entities (i.e: adductor, pubic, inguinal, iliopsoas related GP)

ii. hip-related GP

iii. acute groin injuries

iv. other causes of GP.

During the same Congress have also been defined the other causes of GP set out at point iv. that we report in Table 2.

Injury mechanisms and predisposing factors with particular reference to the football

One of the sports where GP is most frequently is football $^{3}$. Some studies report that the GP incidence in football is equal to 0.6 injuries per 1000 playing hours, 0.3 injuries per 1000 training hours and 1.8 injuries per 1000 match hours 4 .

Many technical movements in football may favor the onset of the injury: jumps, dribbling, cutting movements in general, takles performed sliding with abducted leg and adductor muscle contracted. These are factors that cause high stress on pubis symphysis, triggering a synergic mechanism between adductors and abdominal muscles ${ }^{5}$.

Intrinsic and extrinsic factors may predispose the athlete to the groin pain syndrome. Among the intrinsic factors, those receiving the greater consensus in literature are:

i. hip joint diseases ${ }^{6}$

ii. functional imbalance between abdominal and adductor muscles, with a weakness of abdominal muscles compared to the adductors leading to their excessive stiffness or a weakness of both 
Table 1. The differential diagnosis of groin pain in athletes proposed by Omar et al. ${ }^{1}$ (modified).

\author{
Category 1: Visceral causes \\ Inguinal hernia \\ Other abdominal hernias \\ Testicular torsion
}

\section{Category 2: Hip-associated causes}

Acetabular labral tear and femoroacetabular impingement

Osteoarthritis

Snapping hip syndrome and iliopsoas tendonitis

Avascular necrosis

lliotibial band syndrome

\section{Category 3: Pubic symphyseal causes}

Rectus abdominis strain

Adductor muscle-tendon dysfunction

Rectus abdominis-adductor longus aponeurosis tear

Osteitis pubis

\section{Category 4: Infectious causes \\ Septic arthritis \\ Osteomyelitis}

Category 5: Pelvic inflammatory disease

Prostatitis

Epididymitis and orchitis

Herpes infection

\section{Category 6: Inflammatory causes \\ Endometriosis \\ Inflammatory bowel disease \\ Pelvic inflammatory disease}

\section{Category 7: Traumatic causes}

Stress fracture

Tendon avulsion

Muscle contusion

Baseball pitcher-hockey goalie syndrome

\section{Category 8: Developmental causes}

Apophysitis

Growth plate stress injury or fracture

Legg-Calvé-Perthes disease

Developmental dysplasia

Slipped capital femoral epiphysis

\section{Category 9: Neurologic causes}

Nerve entrapment syndromes (e.g. ilioinguinal nerve)

Referred pain

Sacroiliitis

Sciatic entrapment (piriformis syndrome)

Hamstring strain

Knee pain

\section{Category 10: Neoplastic causes}

Testicular carcinoma

Osteoid osteoma

Table 2. An overview of other condition causing GP in athletes. From Weir (2015) modified.

\begin{tabular}{ll}
\hline Less common & Not be missed \\
\hline Inguinal or femoral hernia & Slipped capital femoral epiphysis \\
Stress fracture: & Perthes' disease \\
-Neck of femur & Intra-abdominal abnormality \\
-Pubic ramus & -Prostatitis \\
-Acetabulum & -Urinary tract infection \\
Nerve entrapment: & -Gynecological conditions \\
-Obturator & \\
-llioinguinal & Spondyloarthropaties \\
-Genitofemoral & - -Ankilosing spondylitis \\
Referred pain & Avascular necrosis of the femur head \\
-Lumbar spine & Tumors \\
-Sacroiliac joint & - Testicular \\
Apophysitis & - Osteoid osteoma \\
-Anterior superior iliac spine & \\
-Anterior inferior iliac spine & \\
-Pubic bone & \\
\hline
\end{tabular}

muscular groups, leading to a reactive contracture of adductor muscles 7,8

iii. adductor weakness ${ }^{4,5}$

iv. limitation of the hip $\mathrm{ROM}^{9}, 10$

v. previous injury $4,9,11$

vi. older age ${ }^{4,12}$.

It is also important to remember that some Authors ${ }^{11}$ proposed as intrinsic cause a core muscular weakness or a delayed onset of trasversus abdominal mus- cle recruitment. Finally it is interesting to note that O'Connor ${ }^{8}$ correlates the adductor longus pathologies with a smaller dominant femur diameter.

The main extrinsic factors that we can find in the literature are:

i. inadequacy of sport equipment: a typical example in football is the use of cleats; too long on dry surfaces or too short on soft ground ${ }^{13}$

ii. inadequate pitch surfaces ${ }^{14-16}$ 
iii. errors in training planification ${ }^{17}$.

Regarding the inadequacy of pitch surfaces we must make some important clarifications. A parameter which we must be carefully assess is represented by the interaction, in terms of mechanical constraint, between the pitch and the shoe. An interesting data in this regard comes from the American National Football League (NFL), which shows that the abductor tendinopathy would increase by $27 \%$ on the artificial turf pitches when compared to natural turf pitches ${ }^{18}$, although these data does not find further confirmation in the literature ${ }^{19,20}$. Also some natural grass surfaces may be a risk factor for the onset of abductor tendinopathy. The association of hot climates and some types of grass having a particularly strong and deep root system create an excessive constraint between the shoe and the ground. Conversely, other types of grass with an insufficient radical apparatus, if used in cold climates would not be able to create a sufficient mechanical constraint between the foot and the playing surface. Both situations could represent a risk factor for onset of adductor tendinopathy especially in athletes with pelvic instability ${ }^{18}$.

\section{The possible association of different pathology into the groin pain syndrome}

During the evaluation of a patient with groin pain, clinicians often focus on a single etiological cause without considering the possible association of multiple causes. Gilmore ${ }^{21}$ introduced the term "groin pain disruption", which indicates how frequently there is a conjoined tendon injury with avulsion from pubic tubercle and an external oblique aponeurosis lesion, or a disjunction between the conjoined tendon and the inguinal ligament. In addition, $40 \%$ of subjects exhibit adductor muscles weakness. Most recently, some authors showed an association between inguinal canal disease, like sport hernia (SE) and femoroacetabular impingement $(\mathrm{FAl})^{22}$ so as to redefine and expand the concept of "groin pain disruption". In effect, a loss of hip ROM, especially in internal rotation, that is a typical clinical signs of patients with $\mathrm{FAl}^{23}$ may be one factor predisposing to the development of $\mathrm{SE}^{24}$.

Furthermore is important to underline that several studies show a reduction in hip muscle strength in subjects with labral pathology ${ }^{25}$ and $\mathrm{FAl}^{26-28}$. In addition Birmingham et al. ${ }^{29}$ reported from a cadaveric study that the pubic symphysis rotational motion is greater in the subjects affected by FAI, leading to an increasing opening of the anterior aspect of pubic symphysis. Moreover others studies report a reduced hip internal rotation, flexion and abduction ROM in subjects suffering $\mathrm{FAI}^{30,31}$.

These impairments in ROM and muscle strength can alter the symphysis arthrokinematics ${ }^{32,33}$ and cause the onset of abductor tendinopathy ${ }^{34,35}$ and to represent a risk factor for abductor injuries ${ }^{36,37}$.

Furthermore we should not forget the fact that the instability of the pubic symphysis deriving from the al- teration of his arthrokinematics $29,32,33$ may be the cause of osteitis pubis onset of which the main risk factor is believed to be pubic symphysis instability ${ }^{1}$. Finally, it is in any case important to note that osteitis pubis is strongly associated with rectus abdominis and/or adductor longus tendinopathy ${ }^{38}$. Therefore, we can potentially to observe the onset of a complex framework dependent on numerous factors linked together by a complex cause/effect relationship.

This frequent association present in many clinical cases and the varied symptom complex that contribute to the determination of the clinical framework, justify and legitimate the use of the term "groin pain syndrome" (GPS $^{39,40}$. It is also clear that this frequent association of different pathology in GPS often makes the definitive diagnosis, and the consequent appropriate management, difficult and multifactorial ${ }^{41-43}$. Following the above and in order to complete the concepts discussed so far, we present in the second part of this study a case-report, in our opinion paradigmatic.

The aim of this case report is to present a clinical case of GPS due to four different diseases, taking into account the frequency of association of different etiological causes and to suggest a systematic diagnostic process of groin pain syndrome.

\section{Case report}

LM, a 25-year-old professional football player, came to us showing GPS. During the past season (2012/13) he was able to take part in competitive events by undergoing NSAID therapy to avoid pain.

The anamnesis revealed that during the sport season of $2011 / 2012$ the patient suffered a II grade lesion of the right adductor longus muscle.

\section{Radiological evaluation}

The MRI showed a severe osteitis pubis (OP), with a large bone marrow edema (with a greater severity to the right side), irregularities of the pubic symphysis and a bilateral adductor longus tendinopathy.

The patient was evaluated with our standard clinical and radiological assessment, which is:

- standard pelvis AP X-ray and "flamingo view"

- pelvis magnetic resonance imaging (MRI) using Thomas Jefferson University Hospital protocol ${ }^{44}$

- inguinal canal dynamic ultrasound (US) and adductor US.

Dynamic pelvis radiograms (Fig. 1) did not show any significant symphysis instability. However, they showed an aspherical deformity of the left femoral head-neck junction as in FAI (CAM type) associated with a slight reduction of the omolateral joint space. The MRI exam (Fig. 2) showed a moderate irregularity of the pubic symphisis articular surface, signal intensity alteration of the near trabecular bone with a slightly greater alteration of the right side, with fluidattenuate inversion recovery (FLAIRE) hyperintensity (case compatible with severe grade OP) associated 


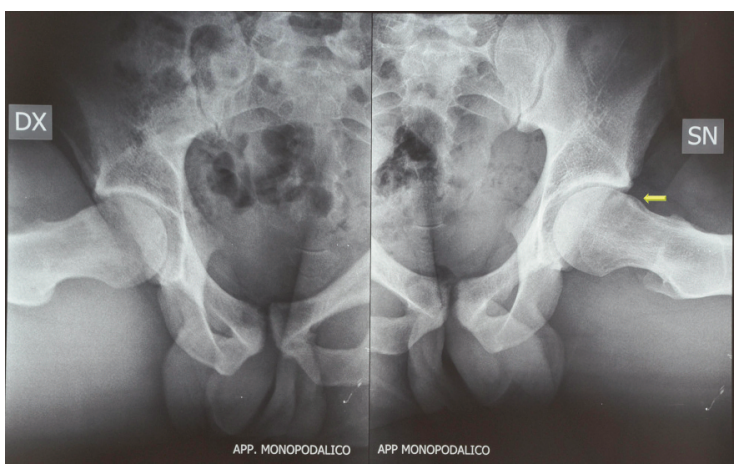

Figure 1. Weight bearing antero-posterior pelvic radiograph shows an aspherical deformity of the left femoral headneck junction (CAM -FAI) associated with a modest reduction in the ipsilateral joint space.

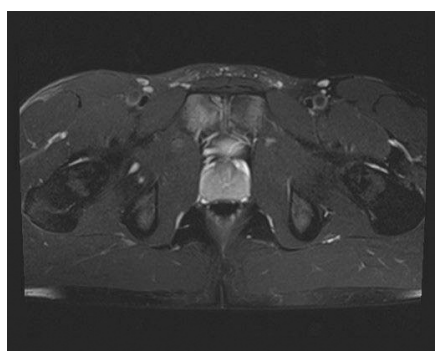

Figure 2. MRI in axial scan STIR DP fat-sat, where it is possible to see how the edema extends to the whole pubic bone bilaterally. This finding is associated with the sign of the "secondary cleft" as a visible curvilinear high signal intensity image adjacent to the antero-inferior surface of the pubic symphysis.

with bilateral adductorial inveterate tendinopathy greater in the left side and "secondary cleft sign".

Significant alterations in signal intensity of the articular surfaces of the hip and the sacroiliac joints were not noticeable. There was no alteration in signal intensity of the adductor belly muscle.

The ecographic examination made with multifrequency linear format, at rest and during abdominal contraction in Valsalva maneuver, is helpful in highlighting direct or indirect signs of herniary pathology. The only limit of this technique stems from the operator's experience level. An ecographic examination and a dynamic examination were performed with the patient supine at rest and confirmed an inveterate tendinopathy of the rectus-adductor region. The dynamic phase, carried out with the examiner's finger in the inguinal canal, showed a bilateral posterior canal wall bulging with anterior convexity, a sign compatible with the presence of sport hernia.

\section{Clinical evaluation}

During clinical examination of the left inguinal-scrotal region, digital pressure on the pubic tuberculus evoked acute and burning pain. While evaluating the

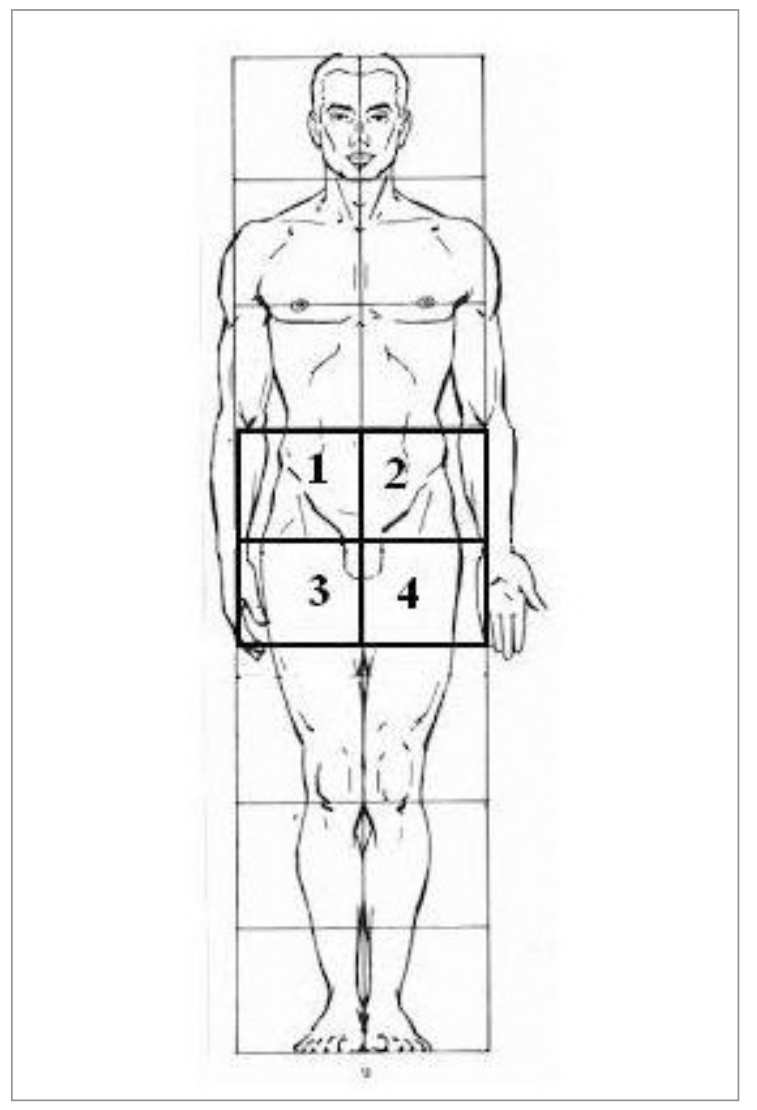

Figure 3. During the dynamometric evaluation, a painful symptom at 3 or 4 quadrant level is suggestive for adductors tendinopathy, while a pain symptom at 1 and 2 level quadrant level is suggestive of an inguinal pathology.

inguinal canal from the superficial orifice (dilated) a weakness was noted in the wall located medial-posteriorly to the epigastric vessels (whose pulse could be distinctly detected). During the Valsalva maneuver the impulse was transmitted to the fingertip, resulting in a clear direct-hernia tumefaction. No signs were found at the testicle and the funiculus elements (M1 as in the European Hernia Society classification) ${ }^{45}$. To the right side there was no algic symptomatology against the pubic tuberculus. The clinical presentation was fundamentally comparable to the contralateral, with medial "bulging" presence during the Valsalva maneuver, and moderate impulse along the funiculus as the finger was leaving the canal $(\mathrm{M} 1+\mathrm{L} 1$ as in the European Hernia Society classification $)^{45}$.

The left hip appeared limited in flexion, with positive FABER and FADDIR test ${ }^{46}$, resulting in a $6 / 10$ algic symptomatology on the VAS scale ${ }^{47}$.

Isometric contraction of adductor muscles with proximal and distal resistance caused a bilateral $7 / 10$ and 9/10 VAS scale value, respectively to quadrant 1 and 2 in Figure 3.

Based on clinical examination and imaging, a diagnosis of pubic osteitis was formulated, associated to bilateral inveterate tendinopathy of the adductor longus muscle, left direct hernia (M1), and left FAI (CAM type). 


\section{Treatment}

Due to the complexity of the medical case as well as the objective difficulty of the conventional treatment for OP, rectus-adductor syndrome (RAS) and FAI (CAM type) with surgical repair with mesh of a bilateral inguinal hernia, it was decided to divide the course of treatment into two distinct phases.

The first phase (F1), which preceded the surgical procedure, lasted three weeks. It consisted of the conventional treatment of OP, RAS and FAI using the following course:

A) Intramuscular therapy based on bisphosphonate with the following modality:

i) sodium clodronate $100 \mathrm{mg}$. pro diem i.m. for a week, $100 \mathrm{mg}$. pro diem i.m. every other day for the next two weeks. The rational of the use of bisphosphonate is based on the fact that they are able to promote the process of resorption of bone marrow edema and then to lower the state of subchondral suffering 48,49 .

B) A Cycle of Extracorporeal Shock Wave Therapy (ESWT), with ecographic readings with these modalities:

i) generator type: electromagnetic, paraboloid cylindrical coil with ecographic in-line control

ii) delivered energy: between 0,05-0,2 $\mathrm{mJ} / \mathrm{mm}^{2}$ depending on the algic symptomatology bearable by the patient during treatment

iii) shots-per-session: 2400

iv) sessions: 3 .

The use of ESWT is justified by their efficacy in the treatment of insertional tendinopathy $50-52$.

C) Application of a nitroglycerin transdermic patch (Deponil $5 \mathrm{mg}$ ) over the area where rectus abdominis and adductor longus muscles meet the pubic symphisis, with this dosage:

i) first 4 days: $1 / 4$ patch from 8 PM to next day's 4 PM

ii) from 4th to 8 th day: $1 / 2$ patch from $8 \mathrm{PM}$ to next day's 4 PM

iii) from 9th day: 1 patch from 8 PM to next day's 4 PM, for 3 weeks.

The mechanism of action of the glyceriyl trinitrate is through nitric oxide (NO), a biologically active metabolite, also called Endothelial Derived Relaxin Factor, showing vasodilatory action. The NO is an important molecular messenger that plays a vital role under many physiological processes ${ }^{53-56}$, including processes of tendon healing ${ }^{57-64}$.

D) Hyaluronic acid infiltration in coxo-femural articulation.

The surgical treatment of a CAM-FAI deformity provide to decompress the prominent bone along the femoral head-neck junction ${ }^{23}$. The outcome of this type of surgical procedure is very satisfactory, in effect some Authors reported that at 6 , and 12 months of follow-up respectively $78 \%$, and $88 \%$ of the professional athletes returned to sport ${ }^{65}$, while another study showed, after 18 months of follow-up, a percentage of professional athletes returning to sport activity equal to $95 \% 66$. However, following the ex- pressed will of the athlete that preferred to avoid surgery, we chose a conservative option. Since the presence of FAl may lead to an increased risk of chondropathy and ultimately hip osteoarthritis $(\mathrm{OA})^{67,68}$ we chose to perform a hyaluronic acid $(\mathrm{HA})$ infiltration in coxo-femural articulation. The rationale for this choice is based on the fact that there is increasing evidence to indicate that clinical efficacy of $\mathrm{HA}$ is mediated through several pathways: anti-inflammatory effects, anti-nociceptive effects, normalization of endogenous HA synthesis and chondroprotection 69-71. Furthermore the slight reduction of the left hip joint space confirmed by X-ray examination further justifies the HA use 69-71. $^{\text {. }}$

E) Cycle of specific FKT based on:

i) strengthening the adductorial muscles through their elongation and detension

ii) strengthening the abdominal muscles, especially the internal and external oblique abdominis, the inferior third of the rectus abdominis, and the transverse abdominis muscle

iii) rebalancing exercises for adductors and abdominal muscles, based on core stability

iv) strengthening of the hip flexors, extensors, abductor and rotators muscles

v) Optimization of hip ROM

The rationale for a cycle of specific FKT is based on the fact that the strengthening of the hip flexors, extensors $^{72,73}$, abductor ${ }^{72,74}$ and rotators muscles ${ }^{75}$ may reduce hip joint loads and the eventual subsequent progression of $\mathrm{OA}^{31}$. Moreover the efficacy of conservative treatment in the recto-abductor tendinopathy is confirmed by the fact that the vast majority of patients respond positively to conservative treatment, both in case of overuse tendinopathy or in muscle-tendon injury ${ }^{76}$.

After the three week duration of $\mathrm{F} 1$, the programmed surgical treatment of a laparoscopic bilateral inguinal hernia repair with mesh was performed.

During this surgical intervention a laparoscopic view discovered a left direct inguinal hernia (M1) (Fig. 4) and a right indirect inguinal hernia (L1, congenital) with a direct component (M1).

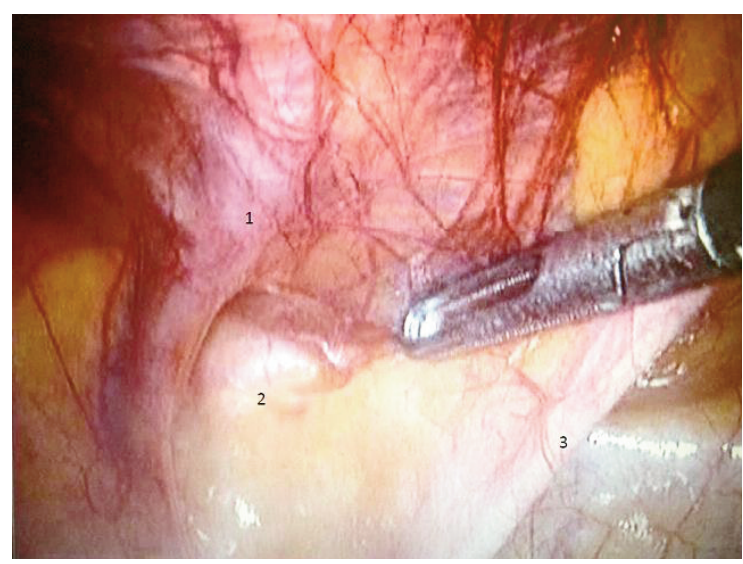

Figure 4. Laparoscopic view of left inguinal region: 1) lateral umbilical ligament; 2) direct hernia; 3) medial umbilical ligament. 
A Laparoscopic Progrip self-fixating mesh (COVIDIEN plc, Ireland) was inserted. This device did not need a mechanical fixation. The clinical post-operatory course was regular.

At discharge, the patient followed a rest period of 7 days because, in this period, the abdomen is distended, painful and treatment is not possible. At the end of this first period, he started the second rehabilitative phase (F2) which consisted in a specific physical therapy protocol (Tab. 3).

F2 was 45 days long. At the end of this period the athlete was gradually reintegrated into the team. He played his first official football match at 71 days after

\section{Table 3. Rehabilitative Protocol.}

Days 1-4

- Cautious mobilization in absence of pain, for a total duration between 10' and 20'

- Walking on treadmill 10'

- Gym bicycle 10'

- Low intensity Core Stability exercises.

Muscular strengthening start (from the second day), following this modality:

- Isometric exercises for the abdominal muscles

- Adductor machine

- Horizontal leg press.

Day 4

- Isometric exercises for the abdominal muscles

- Abductor machine (concentric modality)

- Horizontal leg press

- Standing leg curl (monopodalic execution).

Days 5-14

- Going on with Core Stability exercises

- Going on with isometric exercises for the abdominal muscles, following the previous executive modalities

- Running in water 20'

- Running on treadmill 15 , starting at $8.5-9 \mathrm{~km} / \mathrm{h}$ and progressively increasing velocity to about $80 \%$ of the Maximum Aerobic Speed (MAS)

- Gym bicycle 15'

- Cautious start of basic skipping exercises.

Days 15-28

In addition to the previous program:

- Cautious introduction of plyometric exercises

- Following the strengthening program every other day

- Enhancement of the running program (reaching velocity equal to the MAS, interval training, intermittent training)

- Introduction of running with direction changes

- Cautious introduction of sport-specific training

- Introduction of exercises for the abdominal muscles in concentric modality

- Introduction of exercises for the abdominal muscles in eccentric modality.

Days 29-45

In addition to the previous program:

- Horizontal leg press in explosive modality and monopodalic execution

- Enhancement of plyometric exercises

- Enhancement of sport-specific training

- Introduction of rapidity-velocity-acceleration exercises. surgery (93 days from the beginning of the entire therapeutic course). The duration of the rehabilitation phase conformed to the current literature ${ }^{77}$.

\section{Discussion}

GPS is a clinical problem whose pathophysiology is not yet clear. One reason of this diagnostic difficulty is the anatomical complexity of the groin area and the frequent overlapping of different diseases ${ }^{21}, 22,41-43,78$. In soccer GPS is frequently encountered due to rectusadductors tendinopathy ${ }^{79-81}$ with an incidence of 12$16 \%$ of the whole injuries in a season ${ }^{79}$. This is related to typical soccer movements like jumps, dribbling, rapid twisting and pivoting, long passes, shoots and tackles when the muscles of the lower extremities are in constant abduction with adductors contraction ${ }^{82}$. All these situations inflict high stress to the pubic symphysis, that could start a synergic stress mechanism between adductor and abdominal muscles ${ }^{15,80}$. In addition, kicking and running on inadequate pitch surfaces represents an additional and important stress factor ${ }^{4}, 14-16,18$. It must be emphasized that GPS could arise from acute muscular, tendon or bone injuries, or from an overuse mechanism ${ }^{83}$. Our clinical case can justify the hypothesis that an acute muscular lesion, in this case of the adductor longus muscle, can lead to a functional imbalance of the pubic symphysis. It is important to note that two recent studies show that in football players a reduced ROM of the hip, as in the case of FAI, dramatically increase the risk of abductor injury during kicking movement 36,37 .

This pubic symphysis imbalance can be responsible, in the long run, for both the onset of OP and the clinical manifestation of a previous abdominal wall weakness. This hypothesis is also true in GPS from overuse mechanism or in cases of FAI (both PINCER and CAM FAI), where wrong biomechanics of the hip lead to a functional imbalance of the pubic symphysis. This hypothesis could also be confirmed by two facts: (1) in over $40 \%$ of inguinal pathologies inducing GPS, tendinopathy of the adductor longus muscle is present (2) inguinal canal pathologies, and more specifically SE, are frequently associated to femoroacetabular dysplasia and/or hip impingement ${ }^{22}$.

Obviously the contrary hypothesis could also be true. A primary bone pathology like pubic symphysis osteoarthritis, whose etiology is a continuous microtraumatic mechanism, is an onset to rectus-adductor tendinopathy, or can trigger the clinical manifestation of a previous weakness of the posterior wall of the inguinal canal.

Finally, instability of the pubic symphysis, which causes traction and shearing forces responsible for chronic stresses and imbalance where the muscles insert into the pubic symphysis, can lead to the onset of a bone, muscle-tendon or inguinal GPS.

In our clinical experience GPS is rarely caused by a single disease, as is described in our paradigmatic clinical case. Especially in the case of long-standing GPS in which more types of therapeutic treatment 
have failed, it is advisable to suspect the association of more clinical framework of which one, or more than one, was misunderstood. According to us, it is of extreme importance to evaluate during the diagnostic process the possible association of different etiological diseases, as previously described. The different causes often need various treatments which are difficult to reconcile or, at least, need a different timing. For these reasons, we underline the importance, during the diagnostic process, of an imaging protocol as previously described. This protocol will help confirm, or not, a possible association of bone, muscular, tendon or inguinal diseases, which we observe very frequently in our clinical practice with GPS. Further study is needed to develop a systematic approach to GPS.

\section{Conclusion}

The purpose of this mini-review and correlated case report, that was conduct according to international standards and as required by the Journal ${ }^{84}$, was to underline the concept that in soccer players, GPS is often caused by the association of different diseases. For this reason, we observe a partial resolution of an increasing number of GPS cases, which don't result in a complete athletic recovery. Accordingly, during clinical diagnosis an imaging and clinical evaluation protocol could be useful to obtain an overall view of the different etiological causes of GPS. This clinical case shows that such associations are very frequent and GPS demands a global and deep approach to evaluating this possible coexistence of different clinical frameworks.

\section{References}

1. Omar IM, Zoga AC, Kavanagh EC, et al. Athletic pubalgia and "sports hernia": optimal MR imaging technique and findings. Radiographics. 2008;28(5):1415-1438.

2. Weir A. Report from the groin pain consensus conference 2014. In: Football Medicine Strategies for Player Care (Abstract book). 11th-12th April, 2015. London. 2015:68-69.

3. Hölmich P, Thorborg K, Dehlendorff C, Krogsgaard K, Gluud C. Incidence and clinical presentation of groin injuries in subelite male soccer. Br J Sports Med. 2014;48(16):1245-1250.

4. Engebretsen AH, Myklebust G, Holme I, Engebretsen L, Bahr R. Intrinsic risk factors for groin injuries among male soccer players: a prospective cohort study. Am J Sports Med. 2010;38(10):2051-2057.

5. Benazzo F, Mosconi M, Zanon G, Bertani B. Groin Pain. J. Sport Traumatol Rel Res. 1999;21(1):30-40.

6. Mardones RR, Barrientos CV, Nemtala UF, Tomic A, Salineros UM. Femoroacetabular impingement as a cause of inguinal pain. Rev Med Chil. 2010; 138(1):102-108.

7. Meyers WC, Foley DP, Garrett WE, et al. Management of severe lower abdominal or inguinal pain in high-performance athletes. Am J Sports Med. 2000;28:2-8.

8. O'Connor DM. Groin injuries in professional rugby league players: a prospective study. J Sports Sci. 2004;22:629-636.

9. Gabbe B, Bailey M, Cook J, et al. Hip and groin injuries in young AFL football players-pre-existing or a product of the change to the elite senior level? Br J Sports Med. 2010;44:799802.

10. Verrall GM, Slavotinek JP, Barnes PG, et al. Hip joint range of motion restriction precedes athletic chronic groin injury. J Sci Med Sport. 2007;10:463-466.

11. Maffey L, Emery C. What are the risk factors for groin strain injury in sport? A systematic review of the literature. Sports Med. 2007;37(10):881-894.

12. Arnason A, Sigurdsson SB, Gudmundsson A, et al. Risk factors for injuries in football. Am J Sports Med. 2004;32(Suppl 1):5S-16S.

13. Puig PL, Trouve P, Savalli L. Pubalgia: from diagnosis to return to the sport field. Ann Readapt Med Phys. 2004;47(6):356364.

14. Volpi P. La pubalgie: notre experience. J Traumatol Sport. 1992:9:53-55.

15. Scott AL, Renström FH. Groin injuries in sport. Sport Med. 1999;28(2):137-144.

16. Braun $P$, Jensen $S$. Hip pain - a focus on the sporting population. Aust Fam Phys. 2007;36(6):406-408, 410-413.

17. Paajanen $\mathrm{H}$, Ristolainen $\mathrm{L}$, Turunen $\mathrm{H}$, Kujala UM. Prevalence and etiological factors of sport-related groin injuries in top-level soccer compared to non-contact sports. Arch Orthop Trauma Surg. 2011;131(2):261-266.

18. Kinchington M. Groin pain a view from below. The impact of lower extremity, function and podiatric intervention. ASPETAR Sport Med J. 2013;(2):360-366.

19. Fuller CW, Dick RW, Corlette J, Schmalz R. Comparison of the incidence, nature and cause of injuries sustained on grass and new generation artificial turf by male and female football players. Part 1: match injuries. Br J Sports Med. 2007a;41(Supp I):i20-i26.

20. Fuller CW, Dick RW, Corlette J, Schmalz R. Comparison of the incidence, nature and cause of injuries sustained on grass and new generation artificial turf by male and female football players. Part 2: training injuries. Br J Sports Med. 2007b;41(Suppl l):i27-i32.

21. Gilmore J. Groin pain in the soccer athlete: fact, fiction, and treatment. Clin Sports Med. 1998;17(4):787-793.

22. Nicholson J, Scott M. Conjoint tendon disruption: redifining and recognizing "Golmore's groin" a review of 1200 cases. Hernia. 2012;16:143-240.

23. Pun $\mathrm{S}$, Kumar D, Lane N. Femoroacetabular impingement. Arthritis \& Reumatology. 2015;67(1):17-27.

24. Rambani R, Hackney R. Loss of range of motion of the hip joint. A hypotesis for etiology of sport hernia. Muscles, Ligaments and Tendons Journal. 2015;5(1):29-32.

25. Yazbek PM, Ovanessian V, Martin RL, Fukuda TY. Nonsurgical treatment of acetabular labrum tears: a case series. J Orthop Sports Phys Ther. 2011;41:346-353.

26. Nussbaumer S, Leunig M, Glatthorn JF, Stauffacher S, Gerber $\mathrm{H}$, Maffiuletti NA. Validity and test-retest reliability of manual goniometers for measuring passive hip range of motion in femoroacetabular impingement patients. BMC Musculoskelet Disord. 2010;11:194-204.

27. Casartelli NC, Maffiuletti NA, Item-Glatthorn JF, et al. Hip muscle weakness in patients with symptomatic femoroacetabular impingement. Osteoarthritis and Cartilage. 2011;19:816-821.

28. Hunt MA, Gunether JR, Gilbart MK. Kinematic and kinetic differences during walking in patients with and without symptomatic femoroacetabular impingement. Clin Biomech. 2013;28: 519-523.

29. Birmingham PM, Kelly BT, Jacobs R, McGrady L, Wang M The Effect of Dynamic Femoroacetabular Impingement on Pubic Symphysis Motion. Am J Sports Med. 2012;40:1113-1118.

30. Lamontagne M, Kennedy MJ, Beaulé PE. The effect of cam FAI on hip and pelvic motion during maximum squat. Clin Orthop Relat Res. 2009;467:645-650. 
31. Retchford T, Crossley KM, Grimaldi A, Kemp JL, Cowan SM. Can local muscles augment stability in the hip? A narrative literature review. J Musculoskelet Neuronal Interact. 2013;13:1-12.

32. Neumann DA. Kinesiology of the hip: a focus on muscular actions. J Orthop Sports Phys Ther. 2010;40:82-94.

33. Ward SR, Winters TM, Blemker SS. The architectural design of the gluteal muscle group: Implicati ons for movement and rehabilitation. J Orthop Sports Phys Ther. 2010;40:95-102.

34. Estwanik JJ, Sloane B, Rosemberg MA. Groin strain and other possible causes of groin pain. Physician and Sports Med. 1988;18:59-65.

35. Riley G. The pathogenesis of tendinopathy. A molecular perspective. Rheumatology. 2004;43:131-142.

36. Andersen $\mathrm{L}$. Risk factors for groin injury during football kicking. ASPETAR Sport Medicine Journal. 2014; 3(4):252-256.

37. Tak I, Langhout R. Groin injury in soccer. Step toward a sportspecific approach. From hypothesis to physical examination end treatment. ASPETAR Sport Medicine Journal. 2014;3(4): 272-277.

38. Zoga C. Imaging athletic pubalgia and core muscle injury. Relevance for sports medicine. ASPETAR Sport Med J. 2014;1: 58-65.

39. Vidalin H, Neouze G, Petit J, Brunet-Guedi E. Prise en charge chirurgicale des pubalgies du sportif. J Traumatol Sport. 2004; 21:166-137.

40. Bisciotti GN, Eirale C, Vuckovic Z, Le Picard P, D'Hooge P, Chalabi H. La pubalgia dell'atleta: una revisione della letteratura. Med Sport. 2013;66(1):119-133.

41. Fricker PA. Management of groin pain in athletes. Br J Sports Med. 1997;31:97-101.

42. Bradshaw CJ, Bundy M, Falvey E. The diagnosis of longstanding groin pain: a prospective clinical cohort study. $\mathrm{Br} J$ Sports Med. 2008;42:851-854.

43. Kemp JL, Crossley KM, Schache AG, Pritchard M. Hip-related pain. In: Brukner PD, Bahr R, Blair S, Cook JL, Crossley KM, McConnell J, et al. Clinical Sports Medicine, 4th ed. Sydney, McGraw-Hill 2012:510-544.

44. Khan W, Zoga AC, Meyers WC. Magnetic resonance imaging of athletic pubalgia and the sports hernia: current understanding and practice. Magn Reson Imaging Clin N Am. 2013;21(1): 97-110.

45. Miserez M, Alexandre JH, Campanelli G, et al. The European hernia society groin hernia classification: simple and easy to remember. Hernia. 2007;11(2):113-116.

46. Martin HD, Palmer IJ. History and physical examination of the hip: the basics. Curr Rev Musculoskelet Med. 2013; 6(3):219225.

47. Polly E, Bijur PE, Wendy Silver MA, Gallagher EJ. Reliability of the Visual Analog Scale for Measurement of Acute Pain. Academic Emergency Medicine. 2001;8(12):1153-1157.

48. Gomoll AH, Madry H, Knutsen G, van Dijk N, Seil R, Brittberg $\mathrm{M}, \mathrm{Kon} \mathrm{E}$. The subchondral bone in articular cartilage repair: current problems in the surgical management. Knee Surg Sports Traumatol Arthrosc. 2010;18(4):434-447.

49. Lukas M, Raynauld JP, Martel-Pelletier J, Beaulieu A, Bessette $\mathrm{L}$, et al. Chondroitin sulphate reduces both cartilage volume loss and bone marrow lesions in knee osteoarthritis patients starting as early as 6 months after initiation of therapy: a randomised, double-blind, placebo-controlled pilot study using MRI. Ann Rheum Dis. 2011;70(6):982-989.

50. Wang $\mathrm{CJ}$, Huang $\mathrm{HY}$, Pai $\mathrm{CH}$. Shock wave enhances neovascularization at the tendon-bone junction. J Foot Ankle Surg. 2002;41(1):16-22.

51. Wang CJ, Yang KD, Wang FS, Huang CC, Yang LJ. Shock wave induces neovascularization at the tendon-bone junction. A study in rabbits. J Orthop Res. 2003;21:984-989.

52. Wang CJ. Extracorporeal shockwave therapy in musculoskeletal disorders. J Orthop Surg Res. 2012;7:11.
53. Bult H, Boeckxstaens GE, Pelkmans PA, Jordaens FH, Van Maercke YM, Herman AG. Nitric oxide as an inhibitory nonadrenergic non-cholinergic neurotransmitter. Nature. 1990; 345:346-347.

54. Dusting GJ. Nitric oxide in cardiovascular disorders. J Vasc Res. 1995;32:143-161.

55. Kirkeboen KA, Andreassen AK, Kvernmo HD, Strand OA. Nitric oxide-basal biochemistry and physiological aspects. Tidsskr Nor Laegeforen. 1999;119:4056-4060.

56. $\mathrm{Li} \mathrm{H}$, Forstermann U. Nitric oxide in the pathogenesis of vascular disease. J Pathol. 2000;190:244-254.

57. Murrell GAC, Szabo C, Hannafin JA, et al. Modulation of tendon healing by nitric oxide. Inflamm Res. 1997;46:19-27.

58. Lin J-H, Wang M-X, Wei A, Zhu W, Diwan AD, Murrell GAC. Temporal expression of nitric oxide synthase isoforms in healing Achilles tendon. J Orthop Res. 2001a;19:136-142.

59. Lin J-H, Wang M-X, Wei A, Zhu W, Murrell GAC. The cell specific temporal expression of nitric oxide synthase isoforms during Achilles tendon healing. Inflamm Res. 2001b;50:1-8.

60. Paoloni JA, Appleyard RC, Nelson J, Murrell GAC. Topical nitric oxide application in the treatment of chronic extensor tendinosis at the elbow: a randomized, double-blinded, placebo controlled clinical trial. Am J Sports Med. 2003;31:915-920.

61. Paoloni J, Appleyard R, Murrell GAC. A randomized doubleblind placebo controlled clinical trial investigating the use of topical nitric oxide application in the treatment of Achilles tendonitis. J Bone and Joint Surg Am. 2004;86:916-922.

62. Paoloni JA, Appleyard RC, Nelson J, Murrell GAC. Topical glyceryl trinitrate application in the treatment of chronic supraspinatus tendinopathy: a randomized, double-blinded, placebo-controlled clinical trial. Am J Sports Med. 2005;33:806813.

63. Paoloni J, Murrell GAC. Three-year followup study of topical glyceryl trinitrate treatment of chronic noninsertional Achilles tendinopathy. Foot Ankle Int. 2007;28:1064-1068.

64. Paoloni J. Current Strategy in the Treatment of Achilles Tendinopathy. In: Achilles'tendon. Edited by Andrej Čretnik. Published by InTech Rijeka, Croatia, 2012.

65. Malviya A, Paliobeis CP, Villar RN. Do professional athletes perform better than recreational athletes after arthroscopy for femoroacetabular impingement? Clin Orthop Relat Res. 2013; 471:2477-2483.

66. Byrd JW, Jones KS. Arthroscopic management of femoroacetabular impingement in athletes. Am J Sports Med. 2011;39 (Suppl 1):7S-13S.

67. Lewis CL, Sahrmann SA. Acetabular labral tears. Phys Ther. 2006;86:110-121.

68. Nicholls AS, Kiran A, Pollard TCB, et al. The association between hip morphology parameters and nineteen-year risk of end-stage osteoarthritis of the hip: a nested case-control study. Arthritis Rheum. 2011;63:3392-3400.

69. Migliore A, Martin LS, Alimonti A, Valente C, Tormenta S. Efficacy and safety of viscosupplementation by ultrasound-guided intra-articular injection in osteoarthritis of the hip. Osteoarthritis and Cartilage. 2003;11:305-306.

70. Migliore A, Massafra U, Bizzi E, et al. Comparative, doubleblind, controlled study of intra-articular hyaluronic acid (Hyalubrix $($ ) injections versus local anesthetic in osteoarthritis of the hip. Arthritis Res Ther. 2009;11(6):R183.

71. Colen S, Van den Bekerom MP, Bellemans J, Mulier M. Comparison of intra-articular injections of Hyaluronic Acid and Corticosteroid in the treatment of Osteoarthritis of the hip in comparison with intra-articular injections of Bupivacaine. Design of a prospective, randomized, controlled study with blinding of the patients and outcome assessors. BMC Musculoskelet Disord. 2010;11:264.

72. Boren K, Conrey C, Le Coguic J, Paprocki L, Voight M, Robinson TK. Electromyographic analysis of gluteus medius and 
gluteus maximus during rehabilitation exercises. Int J Sports Phys Ther. 2011;6:206-223.

73. Fisher BE, Lee Y, Pitsch EA, et al. Method for Assessing Brain Changes Associated With Gluteus Maximus Activation. J Orthop Sports Phys Ther. 2013:43:214-221.

74. Cambridge EDJ, Sidorkewicz N, Ikeda DM, McGill SM. Progressive hip rehabilitation: The effects of resistance band placement on gluteal activation during two common exercises. Clin Biomech. 2012;27:719-724.

75. GiphartE, Stull J, LaPrade R, Wahoff M, Philippon M. Recruitment and Activity of the Pectineus and Piriformis Muscles During Hip Rehabilitation Exercises. Am J Sports Med. 2012;40:1654-1663.

76. Atkinson HD, Johal P, Falworth MS, Ranawat VS, Dala-Ali B, Martin DK. Adductor tenotomy: its role in the management of sports-related chronic groin pain. Arch Orthop Trauma Surg. 2010;130(8):965-970.

77. Paaganen H, Brinck T, Hermunen H, Airo I. Laparoscopic surgery for chronic groin pain in athletes is more effective than non-operative treatmen: a randomized clinical trial with magnetic resonance imaging of 60 patients with sportman's hernia (athletic pubalgia). Surgery. 2011;150:99-107.

78. Bouvard M, Dorochenko P, Lanusse P, Duraffour H. La pubal- gie du sportif - stratègie thérapeutique. J Traumatol Sport. 2004;21:146-163.

79. Werner J, Hägglund M, Waldén M, Ekstrand J. UEFA injury study: a prospective study of hip and groin injuries in professional football over seven consecutive seasons. $\mathrm{Br} \mathrm{J}$ Sports Med. 2009;43(13):1036-1040.

80. Ekstrand J, Hägglund $M$, Waldén $M$. Injury incidence and injury patterns in professional football: the UEFA injury study. $\mathrm{Br}$ J Sports Med. 2011;45(7):553-558.

81. Eirale C, Farooq A, Smiley FA, Tol JL, Chalabi H. Epidemiology of football injuries in Asia: a prospective study in Qatar. J Sci Med Sport. 2013;16(2):113-117.

82. Sheen AJ, Stephenson BM, Lloyd DM, et al. 'Treatment of the sportsman's groin': British Hernia Society's 2014 position statement based on the Manchester Consensus Conference. Br J Sports Med. 2014;48(14):1079-1087.

83. Akermark $C$, Johansson $C$. Tenotomy of the adductor longus tendon in the treatment of chronic groin pain in athletes. Am J Sports Med. 1992;20(6):640-643.

84. Padulo J, Oliva F, Frizziero A, Maffulli N. Muscles, Ligaments and Tendons Journal. Basic principles and recommendations in clinical and field science research. MLTJ. 2013;4:250-252. 